\title{
Philonsorbonne
}

11 | 2017

Année 2016-2017

\section{Le primat de la perception dans le concept d'hallucination}

\section{Mathieu FRÈREJOUAN}

\section{(2) OpenEdition}

\section{$\checkmark$ Journals}

\section{Édition électronique}

URL : https://journals.openedition.org/philonsorbonne/849

DOI : 10.4000/philonsorbonne.849

ISSN : 2270-7336

Éditeur

Publications de la Sorbonne

\section{Édition imprimée}

Date de publication : 1 janvier 2017

Pagination : 31-52

ISSN : 1255-183X

\section{Référence électronique}

Mathieu FRĖREJOUAN, « Le primat de la perception dans le concept d'hallucination », Philonsorbonne [En ligne], 11 | 2017, mis en ligne le 06 janvier 2017, consulté le 09 juin 2021. URL : http:// journals.openedition.org/philonsorbonne/849; DOI : https://doi.org/10.4000/philonsorbonne.849 


\title{
Le primat de la perception dans le concept d'hallucination
}

\author{
Mathieu FreREJOUAN
}

\section{Introduction}

Si la philosophie de la perception contemporaine (notamment de tradition analytique) s'intéresse au concept d'hallucination, c'est seulement dans la mesure où ce dernier nous dit quelque chose de la perception. Ainsi, lorsque le philosophe invoque l'hallucination, c'est avant tout parce que, étant à la fois indifférenciable de la perception et indépendante de tout objet externe, elle semble mettre en question la transparence et la réalité de notre expérience perceptive. En d'autres termes, le concept d'hallucination sert principalement à mettre en lumière l'inconsistance $\mathrm{du}$ réalisme naif ${ }^{1}$ qui échouerait à rendre compte du fait que ce que nous percevons peut se révéler n'être qu'une image produite par notre esprit. C'est notamment en réaction à cette menace qu'est née, au sein du réalisme naïf, la théorie dite «disjonctiviste $»^{2}$. Cette dernière s'oppose

\footnotetext{
1. Nous entendons par « réalisme naïf » la théorie d'après laquelle toute expérience véridique «apparaît au sujet comme ayant certaines propriétés parce que le sujet perçoit ces propriétés » (H. Logue, «What should the naïve realist say about total hallucinations ? », Philosophical Perspectives, 26, (2012), p. 173) ou, pour dire les choses autrement, la théorie d'après laquelle ce qui est perçu n'est rien d'autre que l'objet lui-même et ses propriétés.

2. La théorie disjonctiviste est traditionnellement attribuée à Hinton qui, en 1973 dans Experiences, oppose à la thèse d'un élément commun entre perception et illusion la possibilité d'une analyse disjonctive. Selon Byrne et Logue (A. Byrne \& H. Logue, «Either/Or», in Disjunctivism: Perception, Action, Knowledge, A. Haddock \& F. Macpherson (éds.), Oxford, Oxford University Press, 2008) on peut distinguer, à la suite de Hinton, un disjonctivisme épistémologique et un disjonctivisme métaphysique. L'approche épistémologique se trouve notamment chez McDowell (J. McDowell, «Criteria, Defeasibility, and Knowledge », in Disjunctivism, contemporary readings, A. Byrne \& H. Logue, Cambridge, MIT Press, 2009), qui aborde la question des rapports entre hallucination (ou illusion) et perception à partir de ce que le sujet «sait » ou «croit savoir », c'est-à-dire sans se prononcer sur la nature des états
} 
$\mathrm{au}$ « conjonctivisme $»^{3}$ en soulignant le fait qu'on ne saurait soutenir qu'il existe des éléments communs entre l'expérience perceptive et hallucinatoire sous prétexte qu'elles ne peuvent être différenciées l'une de l'autre. En effet, pour toute proposition portant sur une expérience perceptive du type «il me semble voir $x$ », il est possible de proposer une analyse disjonctive qui, en posant qu'il s'agit soit d'une perception véridique soit d'une hallucination, évite la conclusion d'après laquelle l'expérience perceptive et l'expérience hallucinatoire sont des états mentaux de même nature.

Toutefois, ce qui nous intéresse ici ne sont pas tant les arguments qui ont été avancés de part et d'autre, que les présupposés qui permettent à ce débat d'exister. En effet, on notera que conjonctivistes et disjonctivistes ont en commun un seul et même concept d'hallucination, puisque dans un cas comme dans l'autre l'expérience hallucinatoire est considérée comme ne pouvant être différenciée d'une expérience perceptive véridique. Or ce consensus a de quoi surprendre dans la mesure où cette identité entre hallucination et perception, loin d'être évidente, s'est trouvée au cœur d'une question qui a traversé toute l'histoire de la psychopathologie, à savoir ce que peut signifier être une «hallucination véritable ${ }^{4}$. Ainsi, dès l'introduction du concept d'hallucination au début du XIX ${ }^{\text {ème }}$ siècle, les psychiatres se sont demandé de manière récurrente par quels critères il serait pertinent de différencier les «vraies » hallucinations, c'est-à-dire celles qui ont véritablement l'air de perceptions, des «fausses » hallucinations,

mentaux. L'approche métaphysique est avant tout représentée par Martin (M.G.F. Martin, «On being alienated», in Perceptual Experience, T.S. Gendler \& J. Hawthorne (éds.), Oxford, Oxford University Press, 2006) et consiste à nier l'existence d'éléments communs entre le «bon » et le «mauvais » cas, de sorte que perception et hallucination constituent des états mentaux absolument distincts.

3. Le conjonctivisme désigne la théorie philosophique selon laquelle on peut déduire de l'indifférenciabilité entre hallucination et perception l'existence d'éléments communs à ces états. Toutefois, comme cela a été noté par J. Benoist dans Le Bruit du Sensible, l'analyse conjonctive d'une expérience étant un non-sens (un état ne pouvant être une perception $e t$ une hallucination), le «conjonctivisme » n'est pas tant une position philosophique qu'un terme utilisé par le disjonctiviste pour désigner toute théorie qui admet la possibilité d'éléments communs à la perception et l'hallucination. De fait, comme nous le verrons, si la théorie d'une identité de nature entre hallucination et perception repose sur certaines confusions conceptuelles, ces dernières n'ont pas été dissipées mais au contraire renforcées par le disjonctivisme.

4. L'hallucination véritable dont il est question ici renvoie aux hallucinations dont l'apparence est considérée comme authentiquement perceptive. Il ne sera donc pas question des hallucinations véritables au sens épistémique du terme, c'est-à-dire qui correspondent indirectement à des objets externes réels, comme c'est le cas des hallucinations dites «télépathiques» des psychistes (voir à ce propos P. Le Maléfan, «L'hallucination télépathique ou véridique dans la psychopathologie de la fin du XIX ${ }^{\text {ème }}$ siècle et du début du $\mathrm{XX}^{\mathrm{eme}}$ siècle », L'évolution psychiatrique, 73, (2008), p. 15-39) ou des «hallucinations véridiques» que l'on trouve dans certaines expériences de pensée en philosophie où l'expérience sensorielle correspond par accident à l'objet externe tout en ayant pour cause une activité spontanée du cerveau (voir à ce propos D. Lewis, «Veridical hallucination and prosthetic vision », in Vision and Mind: Selected Readings in the Philosophy of Perception, A. Noë \& E. Thompson (éds.), Cambridge, The MIT Press, 2002). 
qui ressemblent à la perception sans jamais se confondre avec elle. Si la plupart d'entre eux s'accordent sur le fait que l'hallucination véritable se reconnait à ce qu'elle est identique à une perception, les opinions divergent toutefois en ce qui concerne le contenu de la «fausse hallucination», laquelle change continuellement de nom («hallucination psychique», «pseudohallucination », « hallucinose », etc.) et de visage.

Le cœur de notre propos sera de montrer que l'indétermination du concept de «fausse hallucination» s'explique par celui de «perception», l'hallucination véritable n'étant finalement rien d'autre que le reflet de ce que chaque psychiatre appelle «percevoir». Or le fait que le concept d'hallucination soit toujours dérivé d'une conception déterminée de la perception remet en question, selon nous, l'usage que prétend en faire la philosophie. On peut en effet se demander comment il est possible de passer du concept psychopathologique d'hallucination, dont le contenu est dérivé $\mathrm{du}$ sens que nous donnons à la perception, au concept philosophique d'hallucination, où c'est cette dernière qui semble déterminer ce que signifie percevoir. Notre hypothèse sera que, loin de pouvoir rendre compte de cette inversion, le concept d'hallucination dans la philosophie de la perception contemporaine part toujours d'une certaine conception de la perception et, par suite, présuppose ce qu'il est censé démontrer.

\section{L'hallucination véritable dans l'histoire de la psychopathologie}

\subsection{L'hallucination psychique}

Pour comprendre la logique à l'œuvre dans la distinction entre vraie et fausse hallucination en psychopathologie, puis en philosophie, il est nécessaire de revenir aux origines du concept d'hallucination. Ce dernier, longtemps cantonné à un emploi marginal au sein de la médecine somatique, ne commence à préoccuper aliénistes et philosophes qu'à partir de 1817, date à laquelle Esquirol lui consacre un article dans le Dictionnaire des sciences médicales de Panckoucke. L'auteur présente alors l'hallucination comme l'état propre à «un homme en délire qui a la conviction intime d'une sensation actuellement perçue, alors que nul objet extérieur propre à exciter cette sensation n'est à portée de ses sens $»^{5}$. L'ambiguïté de cette définition, qui peut signifier soit que l'halluciné a une sensation fausse qu'il croit réelle soit qu'il croit à tort avoir une sensation, se trouve au cœur des débats entre aliénistes. Jules Baillarger joue alors un rôle crucial en suggérant que l'origine du problème vient avant tout du fait que ses contemporains ont «confondu sous le nom commun d'hallucination

5. E. Esquirol, «Hallucination », in Dictionnaire des sciences médicales, Panckoucke (éd.), 1817, vol. 20, p. 1. 
plusieurs phénomènes distincts, quoique semblables en apparence $»^{6}$. Pour mettre fin à cette confusion il propose donc de distinguer l'hallucination véritable, dont le contenu est bien celui d'une perception, des fausses hallucinations, qui s'en écartent. En effet, selon Baillarger, une erreur fréquente du médecin aliéniste est de croire que le patient qui dit entendre des voix se réfère nécessairement à une expérience auditive, alors qu'une analyse attentive du discours de certains d'entre eux montre qu'ils n'entendent pas toujours au sens perceptif du terme :

«Il en est, en effet, qui, comme ils le disent eux-mêmes, n'éprouvent rien qui ressemble à une sensation auditive, ils entendent la pensée. Le phénomène chez eux n'a rien de sensoriel. La voix qui leur parle est une voix secrète, intérieure, et tout-à-fait différente de celle qu' on perçoit par les oreilles »?

Cette observation de Baillarger, dont la pertinence est encore reconnue aujourd'hui, lui permet de mettre en évidence le caractère trompeur du discours des malades. Certes, ces derniers disent «entendre des voix », mais s'ils emploient le terme de « voix » c'est simplement «faute d'un autre qui rend mieux ce qu'ils éprouvent $»^{8}$. Leur expérience ne comporte, en réalité, rien de sensoriel et par suite doit être distinguée des autres hallucinations. C'est pour mettre fin à cette confusion que Baillarger proposera alors de diviser l'hallucination en «psychique » et «psycho-sensorielle » :

« Je crois qu'il y a lieu d'admettre deux sortes d'hallucinations, les unes complètes, composées de deux éléments et qui sont le résultat de la double action de l'imagination et des organes des sens : ce sont les hallucinations psycho-sensorielles; les autres, dues seulement à l'exercice involontaire de la mémoire et de l'imagination, sont tout à fait étrangères aux organes des sens, elles manquent de l'élément sensoriel, et sont par cela même incomplètes : ce sont les hallucinations psychiques ${ }^{9}$.

On notera d'emblée que l'expérience hallucinatoire s'opposant à l'hallucination psychique n'est pas caractérisée comme simplement «sensorielle», comme on pourrait l'attendre, mais comme «psychosensorielle ». Cet ajout d'un élément psychologique à un élément sensoriel vient d'abord du fait qu'il est nécessaire, selon Baillarger, de faire dériver l'hallucination de l'imagination et de la pensée du sujet si l'on veut rendre compte des «rapports de l'hallucination et des idées dominantes des malades $»^{10}$. Néanmoins une autre raison plus fondamentale nous semble commander cette définition, à savoir la volonté non seulement de distinguer

6. J. Baillarger, De l'influence de l'état intermédiaire à la veille et au sommeil sur la production et la marche des hallucinations, Paris, J.-B. Baillière, 1846, p. 275.

7. Ibid., p. 368.

8. Ibid., p. 409.

9. Ibid., p. 309, nous soulignons.

10. Ibid., p. 467. 
mais aussi de hiérarchiser. En effet, tandis que l'hallucination psychosensorielle est «complète », l'hallucination psychique se caractérise par une incomplétude essentielle qui la disqualifie en tant que telle. Cette illégitimité de l'hallucination psychique apparaîtra de manière encore plus claire chez Michéa qui note que de telles expériences doivent porter «le nom de fausse hallucination, et non pas d'hallucination psychique ou intellectuelle, comme la nomme très improprement M. Baillarger ${ }^{11}$. Le reproche qu'adresse ici Michéa à Baillarger vise avant tout le fait que le terme d' « hallucination psychique» peut laisser croire au lecteur qu'il s'agit encore d'une hallucination. C'est pourquoi il lui préfère celui de «fausse hallucination » qui souligne clairement le fait que ces voix entendues seulement en pensée n'ont que l'apparence des « vraies hallucinations ». Cependant, en corrigeant ainsi la manière dont Baillarger nomme ces « voix intérieures » Michéa ne fait que prolonger son projet initial qui est de différencier et hiérarchiser ce que l'on nomme habituellement « hallucination».

La fausseté de ces expériences pose alors la question de savoir au nom de quelle norme de vérité elles sont jugées telles. Cette dernière apparaît clairement dans la définition que donne Baillarger des hallucinations psychiques à la fin de son mémoire. Il s'agit en effet, selon lui, de «perceptions purement intellectuelles, ayant leur point de départ dans l'exercice involontaire de la mémoire et de l'imagination, et qui sont souvent assimilées à tort par les malades aux perceptions sensorielles ${ }^{12}$. Ce que met en évidence cette définition c'est que ces «perceptions purement intellectuelles » ne sont nommées «hallucinations » que dans la mesure où le malade fait parfois l'erreur de les assimiler à des «perceptions sensorielles ». Par suite, c'est avant tout la sensorialité qui constitue le critère décisif permettant de différencier vraie et fausse hallucination. On pourrait alors objecter que si le malade «assimile» son expérience à une perception sensorielle, c'est bien parce que ces dernières ont des propriétés en commun, de sorte que ce critère de différenciation serait relativement flexible. Cependant, selon Baillarger, le malade ne se trompe pas tant sur la nature de son expérience que sur les termes qu'il emploie pour la décrire. En effet, le malade confronté à des hallucinations psychiques se trouve face à une expérience qui n'a rien de commun avec l'expérience quotidienne des sujets sains, de sorte qu'il ne saurait trouver les mots adéquats pour en rendre compte. Baillarger observe alors que pour surmonter cette difficulté, certains vont recourir à des principes occultes comme le magnétisme, la possession d'un sixième sens ou encore la capacité de dialoguer avec les esprits. Mais pour la plupart des malades, «il est bien plus simple de rester dans la règle commune en soutenant qu'on entend des voix extérieures et en tout semblables aux voix ordinaires ${ }^{13}$. L'origine de l'erreur se trouve donc dans l'incapacité du malade à trouver les mots pour décrire son expérience,

11. C.-F. Michéa, Du délire des sensations, Paris, Labé, 1851, p. 113.

12. Op. cit, p. 471, nous soulignons.

13. Ibid, p. 410. 
laquelle le mène à chercher ceux-ci dans le vocabulaire de la perception, dont le médecin fera à tort une interprétation littérale. Toutefois, il ne saurait y avoir de confusion au niveau du contenu même de l'expérience. Ainsi, tandis que l'hallucination psycho-sensorielle est réellement indifférenciable d'une perception sensorielle, l'hallucination psychique ne se confond avec cette dernière que dans le discours du patient et dans l'esprit du médecin.

\subsection{La pseudohallucination}

Jusqu'ici le concept d'hallucination véritable en psychopathologie peut sembler en parfaite cohérence avec le concept philosophique d'hallucination, l'un comme l'autre étant pensés comme indifférenciables d'une perception. Mais, justement, la division entre hallucination psychique et psychosensorielle introduite par Baillarger va être de plus en plus critiquée à partir de la seconde moitié du XIX ${ }^{\text {ème }}$ siècle, notamment par Victor Kandinsky qui propose, dès son article de $1884^{14}$, une nouvelle manière de distinguer fausse hallucination et hallucination véritable. En effet, selon ce dernier ce que Baillarger décrit comme étant des « voix intérieures » ressemble davantage ce que l'on nomme alors de la "pensée forcée », c'est-à-dire ces pensées que le malade a le sentiment de subir indépendamment de sa volonté. Or, selon Kandinsky, les patients ne se réfèrent pas à ces expériences comme relevant de «l'audition » mais plutôt comme relevant du «mental » ${ }^{15}$. Par suite, les phénomènes intellectuels que décrit Baillarger ne sauraient être nommés « hallucinations psychiques», ni même «fausses hallucinations», dans la mesure où même le discours du patient ne peut nous amener à les confondre avec des hallucinations. C'est pourquoi, si une frontière entre vraie et fausse hallucination doit être introduite, selon Kandinsky, il est nécessaire de la tracer au sein même du concept d'hallucination psycho-sensorielle. Et, de fait, ce dernier soutiendra qu'il existe certains cas où ce que l'halluciné éprouve est bien une sensation sans objet, sans être pour autant une « hallucination authentique». Le psychiatre russe propose alors de donner à cette nouvelle sous-catégorie le nom de «pseudohallucination» :

«J'entends par [pseudohallucination] les cas où se présentent à la conscience (...) des images, très vives et extrêmement précises d'un point de vue sensoriel (...), qui cependant se différencient très nettement, pour la conscience réceptrice elle-même, des images véritablement hallucinatoires en ce qu'elles n'ont pas le caractère de réalité objective propre à ces dernières, qu'elles sont au contraire immédiatement reconnues comme quelque chose de subjectif $»^{16}$.

14. V. Kandinsky, «Kritische und Klinische Betrachtungen in Gebiete der Sinnestäuschungen », Centralblatt für Nervenheilkunde, Psychiatrie und gerichtliche Psychopathologie, 7, (1884), p. 481-485.

15. V. Kandinsky, Sur les pseudo-hallucinations, trad. A. Maufras Du Chatellier, Paris, l'Harmattan, 2013, p. 113.

16. Ibid., p. 60. 
Avant d'entrer dans l'analyse de cette description de l'expérience pseudohallucinatoire, il est important de noter à quel point elle s'écarte du critère de différenciation avancé par Baillarger. En effet, il s'agit d'images qui (comme l'hallucination psycho-sensorielle) présentent une sensorialité aussi «vive» et «précise » que celle d'une perception mais qui restent néanmoins subjectives et qualitativement distinctes des hallucinations véritables. En tant que telle, la pseudohallucination est une expérience qui ne peut être pensée dans le cadre théorique posé par Baillarger, où l'expérience du sujet se trouvait nécessairement soit du côté de la perception sensorielle soit du côté de l'intellect. Ce qui fait ainsi l'originalité de Kandinsky, c'est l'introduction d'un nouveau critère de différenciation, à savoir ce qu'il nomme le «caractère de réalité objective » qui est le seul en mesure de distinguer «l'hallucination authentique » de la «pseudohallucination».

$\mathrm{Ce}$ critère de «réalité objective» a fait l'objet de nombreux malentendus dans la mesure où, sorti de son contexte, il apparaît comme relativement vague et ambigu. Ainsi, une erreur commune est de l'interpréter comme désignant le jugement de réalité porté par le sujet sur son hallucination. Cette confusion se trouve notamment chez Kurt Goldstein qui, dans son article de 1908, Zur Theorie Der Hallucination, présente comme faisant partie des «pseudohallucinations » toute expérience hallucinatoire que le sujet est capable de juger irréelle. Or, comme le remarquera Karl Jaspers en 1911 dans Zur Analyse der Trugwahrnehmungen, cette interprétation repose sur un contresens fondamental, car le jugement de réalité n'est simplement pas pertinent lorsqu'il est question de l'hallucination. En effet, selon Jaspers, les hallucinations «se présentent à la conscience et non au jugement ou à l'intellect», de sorte que le caractère d'objectivité est "donné » et non «dérivé d'autres aspects de la conscience $»^{17}$. Autrement dit, l'objectivité est une propriété phénoménale intrinsèque à l'expérience de sorte qu'elle ne saurait dépendre du jugement de réalité qui est relatif au sujet et au contexte. De ce fait, le sujet peut juger une hallucination authentique irréelle s'il a conservé ses capacités intellectuelles et est capable de conclure, par raisonnement, que ce qu'il voit ne peut pas être une perception. Inversement, il n'est pas exclu qu'une pseudohallucination soit jugée réelle malgré son apparence subjective si le sujet est délirant.

Mais si nous savons maintenant que l'objectivité est une propriété phénoménale de l'hallucination authentique, nous ne savons pas davantage ce qu'elle est. Dans un chapitre consacré aux pseudohallucinations visuelles, Kandinsky propose une nouvelle distinction qui éclaire son propos :

«Les pseudohallucinations visuelles appartiennent aussi à l'espace subjectif et ont un champ visuel identique à celui des images mémorielles, mais

17. Cité dans C. Walker, «Form and content in Jaspers' psychopathology », in One century of Karl Jaspers' general psychopathology, G. Stanghellini, T. Fuchs (éds.), Oxford, Oxford University Press, 2013, p. 192. 
ce sont des images qui surgissent spontanément, qui sont très précises, très vives, très achevées d'un point de vue sensoriel (...) Les images visuelles hallucinatoires de la conscience non affaiblie appartiennent à l'espace objectif ; la perception sensorielle subjective se produit ici "avec et en même temps" (...) que les perceptions objectives et possède une valeur identique à celles-ci ${ }^{18}$.

Ce qui apparaît ici, c'est avant tout que le caractère d'objectivité propre à l'hallucination authentique n'est rien d'autre que le fait d'appartenir à «l'espace objectif », caractéristique propre aux perceptions véridiques. Au contraire, les pseudohallucinations, bien que sensorielles et spontanées, appartiennent à un espace «subjectif » qui n'est rien d'autre que l'espace où se déploient les images de la mémoire. Ce qui fait alors l'étrangeté des pseudohallucinations, c'est justement le fait qu'elles soient simultanément sur le plan des images, par leur subjectivité, et des perceptions, par leur sensorialité. Ainsi, à partir d'un exemple récurrent dans son œuvre, Kandinsky explique que si nous faisons l'expérience pseudohallucinatoire visuelle d'un «hussard», ce dernier apparaît « devant le sujet qui voit intérieurement, tout comme lors d'un effort volontaire d'imagination ${ }^{19}$, et de ce fait n'apparaît pas au sein de l'espace objectif des perceptions mais dans un espace subjectif distinct de ce dernier. Cependant, le hussard pseudohalluciné est aussi perçu «d'emblée dans ses moindres détails ${ }^{20}$, de sorte que nous verrons non seulement le shako rouge vif de ce dernier, mais aussi la cocarde se trouvant sur le shako, les traits de son visage et ainsi de suite. Ainsi, malgré sa sensorialité, l'image pseudohallucinatoire reste une image subjective distincte de nos perceptions objectives, et ne peut être considérée comme une hallucination authentique.

On pourrait alors être enclin à comprendre cette appartenance à l'espace objectif comme quelque chose qui viendrait s'ajouter à la sensorialité de l'image pseudohallucinatoire. Ainsi, Kandinsky ne ferait que compléter la définition de Baillarger, en précisant qu'une hallucination est véritable dès lors qu'elle se présente comme indifférenciable d'une perception sensorielle et comme objective. Cependant, inscrire Kandinsky dans la continuité de Baillarger revient à mal comprendre le projet de ce dernier pour qui «l'objectivité » doit être une caractéristique non seulement nécessaire mais suffisante de l'hallucination authentique. Cet aspect de sa pensée apparaît clairement dans le chapitre final de son ouvrage où il critique le psychologue Wilhelm Wundt et le psychiatre Louis-Francisque Lélut pour avoir défendu « l'idée que l'hallucination ne serait rien de plus qu'une image très intense issue de la mémoire ou de l'imagination ${ }^{21}$. Cette conception est inacceptable, selon Kandinsky, car non seulement le fait de conférer la sensorialité à l'image n'en fait pas une perception, mais il est même possible

18. Op. cit, p. 90 , nous soulignons.

19. Ibid., p. 95.

20. Idem.

21. Ibid., p. 187. 
de concevoir la perception sans la vivacité sensorielle. Pour justifier cette affirmation au premier abord surprenante, Kandinsky prend l'exemple d'un dispositif de prestidigitation fréquemment utilisé dans les théâtres de son époque. Ce dernier consiste à placer sur scène une vitre inclinée de manière à ce qu'elle projette devant elle le reflet de l'associé du prestidigitateur, qui reste pendant ce temps à l'abri des regards du public. Ce reflet, avec lequel le prestidigitateur fait mine d'interagir, apparaît alors au spectateur comme une image pâle et transparente de l'associé qui a ainsi l'apparence d'un « fantôme ». Or, selon Kandinsky, « le fantôme pâle aura, pour la perception des spectateurs, absolument le même caractère d'objectivité que l'image du prestidigitateur lui-même ${ }^{22}$ de sorte que "l'énorme différence de vivacité et en précision des deux perceptions visuelles n'empêche pas les deux images d'être objectives au même degré ${ }^{23}$. Par suite, pour qu'une image hallucinatoire ait l'apparence d'une perception il n'est pas nécessaire qu'elle soit sensorielle, il faut et il suffit qu'elle fasse partie de l'espace objectif. Inversement, peu importe la vivacité et le détail d'une image pseudohallucinatoire sensorielle, celle-ci ne pourra jamais se faire passer pour une perception véridique.

En substituant ainsi le critère de l'objectivité à celui de la sensorialité Kandinsky ne se contente donc pas de préciser la distinction introduite par Baillarger entre fausse et véritable hallucination, il en change entièrement le sens. Et pourtant ce dernier semble partager la même conception de l'hallucination véritable, à savoir celle d'une expérience indifférenciable d'une perception et pourtant sans objet externe. En effet, l'hallucination authentique pour Kandinsky désigne d'abord et avant tout les «états de conscience qui sont ou bien tout à fait équivalents à des perceptions sensorielles objectives normales ou bien, en l'absence de celles-ci, susceptibles de se substituer à elles $»^{24}$. Ainsi, l'un comme l'autre conçoit l'hallucination véritable comme indifférenciable de la perception tout en proposant des descriptions radicalement différentes de cette dernière. Cependant, le fait que Kandinsky puisse changer les critères de différenciation entre vraie et fausse hallucination, sans altérer la définition originelle de l'hallucination, n'a rien d'étonnant dès lors qu'on s'autorise à redéfinir la perception en termes d'objectivité plutôt que de sensorialité. Ainsi, on observe déjà à travers cette évolution comment le concept d'hallucination véritable semble avant tout varier en fonction de ce que le psychiatre entend par perception. Mais on ne peut prendre la mesure de cette corrélation entre hallucination et perception, et son impact sur la philosophie, qu'en se tournant vers une nouvelle différenciation opposant cette fois hallucination véritable et hallucinose.

22. Ibid., p. 191.

23. Idem.

24. Ibid., p. 186, nous soulignons. 


\subsection{L'hallucinose}

À la suite de l'hallucination psychique et de la pseudohallucination, une nouvelle sous-catégorie apparaît au début du $\mathrm{XX}^{\mathrm{e}}$ siècle : «l'hallucinose ». Ce concept, introduit par Henri Claude et Henri Ey, va marquer durablement l'école de psychopathologie française dans la mesure où il implique de remettre en cause toutes les descriptions précédentes. Ce nouveau changement de paradigme, proposé pour la première fois dans un article coécrit par Claude et Ey en 1932 - Hallucinose et hallucination, les théories neurologiques des phénomènes psycho-sensoriels - se présente d'abord comme un retour aux sources, c'est-à-dire à la définition esquirolienne de l'hallucination. En effet, comme le rappellent ces derniers, pour Esquirol l'état d'hallucination est la «conviction intime d'une sensation actuellement perçue $»^{25}$. Or, comme nous l'avons vu, un des sens possibles de cette définition est que le sujet «croit» en l'existence de ce qu'il dit percevoir sans nécessairement en faire l'expérience sensorielle. Ainsi, «nous conformant à la vraie définition primitive de l'hallucination qui en fait une croyance dans la réalité d'un objet qui n'existe pas », expliquent Claude et Ey, «pour nous la «perception sans objet » », implique l'attribution d'un caractère de réalité à un objet entièrement fictif ${ }^{26}$. C'est en cohérence avec cette définition que Claude et Ey peuvent alors présenter l'hallucinose comme toute expérience se caractérisant par « la présence dans le champ de la conscience d'une sensation ou d'une forme ${ }^{27}$ mais » à laquelle le sujet n'ajoute pas foi $\gg^{28}$. De même que pour Kandinsky l'objectivité était un critère nécessaire et suffisant de la différence entre hallucination authentique et pseudohallucination, de même la croyance en la réalité de l'objet va suffire à distinguer l'hallucination véritable de l'hallucinose.

Cependant, le sens profond de ce retour aux origines ne peut apparaître dans les limites de l'article de 1932, de sorte qu'il est nécessaire de se tourner vers l'analyse approfondie qu'en fera Ey en 1973 dans son Traité des hallucinations. Dans le chapitre consacré à l'histoire des hallucinations, ce dernier affirme que pour Esquirol «les concepts d'Hallucination, de délire, de psychique et de cérébral étaient synonymes ${ }^{29}$, de sorte qu'en définissant l'état d'hallucination comme un état de «conviction intime », il ne faisait rien d'autre que le rapporter à un état de « délire». Mais, selon Ey, ce lien de l'hallucination au délire va être peu à peu oublié par les cliniciens de l'époque (notamment Baillarger) qui, sous «l'empire des schèmes sensationnistes » de la philosophie ambiante, ont conçu l'hallucination

\section{Op. cit, p. 1.}

26. H. Claude, H. Ey, «Hallucinose et hallucination. Les théories neurologiques des phénomènes psychosensoriels », Encéphale, XXVII, 7, (1932), p. 576.

27. Ibid., p. 577.

28. Idem.

29. H. Ey, Traité des hallucinations, Paris, Masson et $C^{\mathrm{ie}}$, 1973, p. 81. 
comme un «phénomène primitivement sensoriel $»^{30}$. Si cette interprétation presque intellectualiste de la théorie esquirolienne de l'hallucination nous paraît historiquement contestable ${ }^{31}$, elle est cependant révélatrice du principe qui guide la pensée d'Ey. En effet, ce qui permet de différencier hallucination véritable et hallucinose, c'est avant tout la notion de délire et non celle de sensation contrairement à ce qu'ont pu croire ses prédécesseurs. Ainsi, la thèse fondamentale d'Ey, dans le Traité des hallucinations, sera que tout état hallucinatoire véritable surgit sur fond de délire et n'est jamais, en tant que tel, premier. Mais cette thèse était déjà présente dans l'article de 1932 de Claude et Ey, dont l'objectif principal est de démontrer que « la conviction intime de percevoir un objet absent exige un trouble important de l'activité psychique $»^{32}$, c'est-à-dire est nécessairement précédée d'un état délirant.

On peut alors se demander si ce nouveau concept d'hallucination véritable est toujours pensé en relation avec la perception. En effet, nous avions vu que si Baillarger et Kandinsky étaient en désaccord sur ce qui constitue une fausse hallucination, ils s'accordent néanmoins à reconnaître qu'une hallucination se reconnaît au fait qu'elle est identique à une perception. De ce point de vue, il semblerait bien que Claude et Ey, en se référant au délire, rompent le lien entre hallucination et perception afin de mettre la croyance au premier plan. En réalité, une telle perspective n'est pas envisageable car, comme ceux-ci le rappellent à de nombreuses reprises, l'hallucination est et reste pour eux une «perception sans objet». On pourrait alors être tenté de voir là une contradiction, la perception et la croyance ne pouvant définir simultanément l'hallucination véritable. Pour lever cette opposition, il est nécessaire de comprendre ce que "percevoir » signifie pour Claude et Ey. Or si on ne trouve aucune définition de la perception dans l'article en question, Ey y consacrera cependant un deuxième article, quelques mois plus tard, intitulé «La croyance de l'halluciné ». Critiquant le concept sensualiste de l'hallucination soutenue

\section{Ibid., p. 82 .}

31. En effet, si pour Esquirol comme pour Ey l'hallucination est bien indissociable du «délire », il n'en reste pas moins que pour ce dernier « un homme est en délire lorsque ses sensations ne sont point en rapport avec les objets extérieurs, lorsque ses idées ne sont point en rapport avec ses sensations» de sorte que «les hallucinations sont la cause la plus fréquente du délire » (E. Esquirol, «Délire», in Dictionnaire des sciences médicales, Panckoucke (éd.), 1814, vol. 8, p. 251). De ce fait, Esquirol semble d'une part associer l'hallucination à la sensation (ce qui exclut la possibilité d'une hallucination purement intellectuelle) et d'autre part concevoir cette dernière comme une cause du délire c'est-à-dire comme étant logiquement indépendante de ce dernier. Par suite il semble difficile de faire du médecin aliéniste un précurseur d'une conception intellectualiste de l'hallucination. On notera cependant qu'il existe un écart, voire des tensions, entre la manière dont Esquirol explique la relation entre délire et hallucination dans son article de 1814 puis dans celui de 1817 , de sorte que l'on peut considérer que l'auteur oscille parfois entre une conception intellectuelle et sensorielle de l'hallucination.

32. Op. cit, p. 615. 
par le psychiatre Pierre Quercy, Ey remarque très justement que «qu'on le veuille ou non, poser le problème de l'hallucination, c'est, du même coup, formuler une opinion sur le mécanisme de la perception ${ }^{33}$, et, par suite, souligne l'importance de définir au préalable ce que signifie percevoir. Il propose alors de préciser ce qu'il entend par perception en opposant celle-ci à la sensation :

«Nous éprouvons une sensation et nous percevons des objets. Éprouver une sensation, c'est avoir conscience d'une impression sonore, olfactive, douloureuse, chaude, froide, etc. Une sensation est une affection. Percevoir, c'est attribuer les impressions sensorielles à un objet, c'est le situer dans la réalité objective (...) Percevoir sans objet, c'est donc intégrer dans la réalité quelque objet qui n'en vient pas $»^{34}$.

Cette conceptualisation de la perception nous permet alors de comprendre comment l'hallucination délirante, pour Claude et Ey, n'a de sens qu'à travers leur référence à la perception. Tout d'abord la sensation est conçue comme une "affection », c'est-à-dire comme ce qui est éprouvé subjectivement par le sujet et qui par suite ne possède pas de réalité propre. $\mathrm{Au}$ contraire, la perception ne place pas le sujet dans un état de réceptivité, mais est un acte qui consiste à «attribuer les impressions sensorielles à un objet » c'est-à-dire à leur conférer une forme d'objectivité. Cependant, cette objectivité ne doit pas être confondue avec celle décrite par Kandinsky. En effet, comme l'a montré Jaspers, l'objectivité chez Kandinsky est une propriété phénoménale de la perception, indépendante du jugement de réalité porté par le sujet sur celle-ci. En revanche, pour Ey, si l'objectivité de l'hallucination ne relève pas du simple jugement, elle n'est pas davantage une dimension phénoménale de la perception, mais appartient plutôt à la dimension active et cognitive de la perception qui consiste à « situer dans la réalité objective ${ }^{35}$ l'objet visé. En ce sens, Ey introduit bien un nouveau critère, qui n'est ni celui de la sensorialité, ni celui de l'objectivité, mais celui de la «réalité » de l'hallucination, au sens où elle est posée comme réelle par la croyance du sujet.

De ce fait, une hallucination sera véritable ou fausse selon qu'elle implique cette croyance ou non. Si le sujet est affecté par une sensation, comme dans l'hallucination psycho-sensorielle de Baillarger, et que cette sensation lui apparaît au sein de l'espace objectif, comme dans l'hallucination authentique de Kandinsky, mais qu'il n'attribue pas cette sensation à un objet réel, alors il ne s'agit pas à proprement parler d'une hallucination véritable. Ainsi, de nombreux cas que leurs prédécesseurs

33. H. Ey, «La croyance de l'halluciné (à propos des études de M. QuERCY sur l'hallucination) », Annales médico-psychologiques, 11, (1932), p. 15.

34. Idem.

35. Idem. 
auraient considérés comme des hallucinations véritables seront considérés par Claude et Ey comme de simples hallucinoses. Inversement, si le patient se réfère aux voix qu'il entend comme à des sujets s'adressant réellement à lui, la réalité qu'il attribue à celles-ci suffit à les caractériser comme des hallucinations véritables indépendamment de leur contenu phénoménal. Cela ne signifie pas pour autant qu'il faille rejeter toute présence de la sensation dans l'hallucination véritable, mais seulement que celle-ci devient secondaire voire indifférente à sa définition. En effet, comme le remarque Ey, la question de la sensorialité est une question «qui importe au psychiatre, mais qui généralement n'intéresse pas du tout le malade. Ce qui l'intéresse - ce n'est pas comment et pourquoi il a des voix! - c'est d'écouter "ce qu'ils disent" " ${ }^{36}$. Ainsi, le clinicien doit davantage se préoccuper de la réalité que le sujet attribue aux voix, voire de leur contenu sémantique, qu'aux propriétés phénoménologiques de ces dernières qui sont des caractéristiques contingentes de l'expérience hallucinatoire. C'est pourquoi l'hallucination véritable continue à être pensée par Ey dans sa relation à la perception, bien que sa signification soit radicalement différente de celle que lui attribuaient Baillarger et Kandinsky.

\subsection{Le primat de la perception dans le concept d'hallucination véritable}

Nous avons ainsi vu à travers trois figures de la fausse hallucination l'hallucination psychique, la pseudohallucination et l'hallucinose - ce qui légitimait et par là donnait son contenu au concept d'hallucination véritable. En effet, par-delà la variation des critères avancés par les auteurs - la sensorialité, l'objectivité, la réalité -, le concept d'hallucination reste en un sens invariable dans la mesure où il se définit toujours par rapport à la perception véridique. Ainsi, la cause des transformations historiques subies par le concept d'hallucination en psychopathologie n'est pas tant à chercher du côté de l'expérience hallucinatoire que du côté de l'évolution des représentations que se font les psychiatres de la perception. Cela ne signifie pas que leur expérience - clinique ou personnelle - de l'hallucination n'ait pas un impact sur leur manière de concevoir la perception, mais que, d'un point de vue conceptuel et logique, c'est bien cette dernière qui constitue le critère de différenciation entre vraie et fausse hallucination. En ce sens, l'histoire de la psychopathologie nous semble mettre en évidence le primat et l'antériorité de la perception dans la conceptualisation de l'hallucination. Or, cette même primauté de la perception remet en question, selon nous, la manière dont la philosophie de la perception fait usage du concept d'hallucination.

36. Ibid., p. 35 . 


\section{L'hallucination parfaite du philosophe}

\subsection{L'argument de l'hallucination}

Notre objectif, dans ce second moment, sera donc de voir comment la philosophie rend compte de cette antériorité du concept de perception par rapport à celui d'hallucination. S'il nous semble possible d'interroger la philosophie à partir de l'histoire de la psychopathologie, c'est d'abord parce que la manière dont celle-là conçoit l'hallucination est loin d'être étrangère au concept des psychiatres. Prenons pour exemple la définition qu'en propose Foster dans The Nature of Perception :

«Dans le cas des hallucinations, ou du moins celles qui nous concernent ici, le sujet a une expérience qui est subjectivement identique à la perception d'un objet physique, quoiqu'il n'y ait aucun objet physique perçu - une expérience qui n'est pas physiquement perceptuelle, mais qui est introspectivement indifférenciable d'une expérience qui l'est $»^{37}$.

Ce qui semble avant tout caractériser l'hallucination pour le philosophe ${ }^{38}$, c'est le fait qu'elle soit indifférenciable d'une perception véridique. Or, comme nous l'avons vu, cette propriété est aussi celle qui caractérise l'hallucination véritable en psychopathologie, de sorte qu'aucun écart conceptuel ne semble apparaître ici. Bien plus, Foster n'est pas loin luimême d'introduire une différence entre vraie et fausse hallucination, puisqu'il précise bien que seules les hallucinations subjectivement indifférenciables d'une perception «nous concernent», ce qui est une manière d'écarter du concept d'hallucination la diversité des expériences hallucinatoires qui s'approchent de la perception sans se confondre avec elle. En ce sens, philosophie et psychopathologie s'accorderaient à reconnaître que l'hallucination psychique, la pseudohallucination ou l'hallucinose, ne sont pas des hallucinations à proprement parler. Toute la question est alors de savoir si cette proximité conceptuelle implique que le philosophe, comme le psychiatre, doit admettre que pour penser l'hallucination il faut d'abord savoir ce que signifie percevoir.

37. J. Foster, The Nature of Perception, Oxford, Oxford University Press, 2000, p. 7 [notre traduction].

38. Même si certains auteurs remplacent le terme d'objet «physique» par celui d'objet « matériel » (A. Byrge \& H. Logue, op. cit., p. 2), « indépendant de l'esprit » (M.G.F. Martin, op. cit., p. 2) ou encore «externe» (H. Robinson, «The Failure of Disjunctivism to Deal with "Philosophers' Hallucinations" ", in Hallucination: Philosophy and Psychology, F. Macpherson \& D. Platchias (éds.), Cambridge, The MIT Press, 2013), cette définition peut être généralisée à l'ensemble de la philosophie de la perception de tradition analytique dans la mesure où ces différentes dénominations sont souvent considérées comme synonymes par les auteurs qui les emploient. Cette variation pour le moins problématique est symptomatique, selon nous, de l'absence d'analyse approfondie du concept d'hallucination. 
Pour répondre à cette question, il est nécessaire de s'intéresser non plus à la manière dont le philosophe définit le concept d'hallucination, mais à l'usage qu'il en fait. En effet, le concept philosophique d'hallucination n'est jamais interrogé pour lui-même, comme ce peut être le cas en psychopathologie, mais n'attire l'attention des philosophes que dans la mesure où il joue un rôle central dans ce que l'on nomme communément «l'argument de l'hallucination». Ce dernier est apparu en philosophie analytique au début du $\mathrm{XX}^{\mathrm{e}}$ siècle, notamment chez des auteurs défendant la théorie des sense-data contre les conceptions réalistes de la perception ${ }^{39}$. C'est sa structure qui va nous permettre de comprendre la fonction qu'occupe ici le concept d'hallucination. Istvan Aranyosi en propose une formulation suffisamment générale pour être représentative des différentes versions de l'argument :

(1) Des hallucinations subjectivement indifférenciables des perceptions véridiques sont possibles.

(2) Si deux états subjectifs sont indifférenciables, ils ont alors une nature commune.

(3) Les contenus des hallucinations sont des images mentales et non des objets concrets externes.

(4) Par suite, les contenus des perceptions véridiques sont des images mentales et non des objets externes concrets ${ }^{40}$.

La première chose qu'il convient de noter est que les relations que cet argument instaure entre hallucination et perception sont symétriquement opposées à celles que nous avons pu observer en psychopathologie. En effet, en psychopathologie, l'indifférenciabilité de l'hallucination et de la perception permettait de déterminer la nature de l'expérience hallucinatoire. C'est ainsi qu'Ey, entre autres, soutenait que dans la mesure où la perception est l'acte de poser un objet comme réel, l'hallucination véritable (qui est identique à celle-ci) se caractérise par la croyance du sujet en sa réalité. Au contraire, l'argument philosophique part du concept d'hallucination comme « image subjective », et c'est l'indifférenciabilité de l'hallucination et de la perception qui va permettre de conclure que la perception est une «image subjective ». De même, le fait que le concept de perception soit déterminé dans la conclusion et non dans la prémisse de l'argument implique qu'il possède un statut différent de celui employé en psychopathologie. Tandis que le psychiatre part d'un concept déterminé de la perception (comme

\footnotetext{
39. L'argument de l'hallucination est évidemment bien plus ancien si l'on le situe dans la continuité de l'argument sceptique classique de l'illusion. Néanmoins, comme nous le montrerons en 2.2, par-delà la filiation historique qui les relie l'un à l'autre, ces arguments ne peuvent être confondus entre eux.

40. I. Aranyosi, «Silencing the Argument from Hallucination», in Hallucination: Philosophy and Psychology, F. Macpherson \& D. Platchias (éds.), Cambridge, The MIT Press, 2013, p. 255 [notre traduction].
} 
sensorielle, objective ou réelle) afin de différencier la vraie de la fausse hallucination, le philosophe part d'un concept indéterminé de la perception et entend en découvrir les propriétés grâce à l'hallucination.

Ainsi, au premier abord, l'usage philosophique du concept d'hallucination apparaît comme paradoxal, sinon contradictoire, dans la mesure où il prétend nous apprendre quelque chose de la perception alors qu'il ne peut se comprendre sans se référer à cette dernière. Néanmoins, il semble possible de réhabiliter cet argument en notant que le psychiatre et le philosophe se réfèrent en réalité à des dimensions différentes de l'hallucination. De fait, les termes d' « image » ou de «subjectivité » ne sont pas utilisés dans le même sens dans la proposition (3) de l'argument ou, par exemple, dans les descriptions de Kandinsky. En effet, pour le psychiatre, ces termes ont pour fonction de décrire les propriétés phénoménales de l'hallucination (et notamment de la pseudohallucination). On ne peut en revanche donner un sens phénoménologique à l'expression d' «image subjective » lorsqu'il s'agit du concept philosophique d'hallucination, dans la mesure où d'un point de vue phénoménal celle-ci est par définition indifférenciable d'une perception. Ce que veut signifier en réalité cette référence à l'imagerie mentale n'est rien d'autre que l'absence de relation entre hallucination et réalité objective. Le statut d'image subjective ainsi attribué à l'hallucination renvoie au statut ontologique de l'expérience et reste entièrement étranger à la manière dont celle-ci est vécue par le sujet. De ces distinctions, on peut alors conclure que, tandis que la psychopathologie tente d'élaborer une description phénoménologique de l'hallucination, la philosophie en détermine les propriétés ontologiques.

Toutefois, cette indépendance de la philosophie vis-à-vis de la phénoménalité de l'hallucination ne va pas de soi. Certes, en un sens, la seule propriété phénoménale que l'argument cité plus haut attribue à l'hallucination est celle d'être «subjectivement indifférenciable» d'une perception. Par suite, dans la mesure où tout ce que le philosophe pourrait dire de l'apparence de l'hallucination ne serait que la répétition de ce qu'il dit déjà de la perception, il semble possible de mettre entre parenthèses toute phénoménologie de l'hallucination. Mais cette solution a un prix, à savoir présupposer qu'une expérience hallucinatoire est en mesure de reproduire à l'identique toutes les propriétés phénoménales de la perception. Or ce présupposé est proprement inconcevable d'un point de vue psychopathologique. En effet, lorsqu'un psychiatre présente une hallucination comme "véritable », il ne s'agit pas pour lui d'affirmer que l'hallucination possède toutes les propriétés de la perception, mais uniquement la propriété essentielle, celle qui est constitutive de l'acte de percevoir. Ainsi, l'hallucination véritable peut être, pour Baillarger, sensorielle sans être tenue pour réelle, comme elle peut être objective, pour Kandinsky, ou l'objet d'une croyance, pour Ey, sans être sensorielle. En ce sens, l'indifférenciabilité de l'hallucination et de la perception en psychopathologie n'implique pas une identité absolue, mais uniquement le partage d'une propriété considérée comme essentielle. Par suite, pour 
que la philosophie puisse mettre entre parenthèses la question de la phénoménologie de l'hallucination, et le primat de la perception qui lui est associé, il est nécessaire qu'elle passe d'une indifférenciabilité relative entre hallucination et perception à une identité absolue des expériences en question.

\subsection{L'hallucination comme possibilité métaphysique}

Cette identité phénoménale entre hallucination et perception, sans laquelle l'argument de l'hallucination n'a plus de sens, a déjà été pressentie comme problématique. En effet, comme le souligne Tim Crane dans Elements of the Mind, l'expérience de l'hallucination est communément associée à un état de conscience pathologique qui ne saurait être comparé à la perception du sujet normal. Par suite, on pourrait objecter que «l'idée séduisante d'une hallucination qui serait indifférenciable d'une expérience perceptive authentique " ne serait rien d'autre qu'une «spéculation empirique illégitime ${ }^{41}$. Et pourtant, selon lui, cette objection n'a pas de quoi inquiéter le philosophe. En effet, l'idée d'une hallucination indifférenciable d'une perception n'est rien d'autre qu'une "possibilité métaphysique ${ }^{42}$ qui, en tant que telle, est entièrement indépendante de la réalité de l'expérience hallucinatoire. Et, de fait, il est fréquent que les philosophes distinguent ainsi leur concept d'hallucination, en le désignant par les termes d' 'hallucination parfaite ${ }^{43}$ ou $d^{\prime}$ ' hallucination du philosophe $»^{44}$, de l'expérience hallucinatoire réelle. On peut alors considérer que psychiatres et philosophes s'intéressent non seulement à des aspects différents de l'hallucination, mais de manière plus radicale qu'ils se réfèrent à des concepts qui sont étrangers l'un à l'autre: tandis que l'indifférenciabilité de «l'hallucination véritable»du psychiatre renvoie à l'existence d'une propriété phénoménale commune, l'indifférenciabilité de «l'hallucination parfaite» du philosophe renvoie à la possibilité métaphysique d'une identité phénoménale totale.

Cependant, cette sortie hors de la réalité empirique ne met pas fin aux problèmes posés par le concept philosophique d'hallucination. Pour comprendre les difficultés inhérentes au concept métaphysique d'hallucination, il est nécessaire de faire un détour par l'argument de l'illusion auquel il est lié historiquement. Ce dernier possède la même structure que l'argument de l'hallucination, à cette différence près qu'il s'agit d'interroger l'indifférenciabilité qui se manifeste entre une perception et une illusion. En effet, tandis que l'hallucination est une expérience

41. T. Crane, Elements of Mind, Oxford, Oxford University Press, 2001, p. 133.

42. Idem.

43. M.G.F. Martin, «The Limits of self-awareness », Philosophical Studies, 120, (2004), p. 37-49.

44.H. Robinson, op. cit., p. 18. 
indépendante de toute réalité externe, l'illusion est la perception erronée ou déformée d'une réalité externe. Ainsi, pour reprendre un exemple classique, si je perçois une tour carrée qui me semble ronde, je ne suis pas en train d'halluciner dans la mesure où je perçois bien une tour, mais je suis en revanche victime d'une illusion puisque je me trompe sur sa forme. Si cette différence conceptuelle a souvent été relevée en philosophie, une autre différence plus fondamentale est que dans le cas de l'illusion, l'argument se réfère toujours à des faits phénoménologiques. En effet, qu'il s'agisse de la tour carrée, du bâton plongé dans l'eau, ou des lignes de Müller-Lyer, tous ces exemples renvoient à une expérience perceptive accessible à tous et dont le mode d'apparaître peut-être décrit. En ce sens, l'argument de l'illusion se présente comme un défi adressé au philosophe réaliste qui doit être capable de rendre compte de ces faits qui font partie, de manière incontestable, de notre expérience perceptive. Or un glissement problématique semble s'être opéré avec le concept d'hallucination. En effet, on exige du philosophe de la perception qu'il rende compte de l'existence de l'hallucination alors que, à la différence de l'illusion, son concept est une pure possibilité métaphysique qui ne s'instancie jamais dans aucune de nos expériences. Par suite, autant il est compréhensible qu'une philosophie de la perception doive rendre compte de l'ensemble des faits perceptifs - réels et illusoires -, autant on peut s'interroger sur la place qu'elle doit accorder à ce qui est seulement métaphysiquement concevable. En ce sens, l'argument de l'hallucination ne saurait avoir un statut équivalent à celui de l'illusion tant qu'il n'aura pas donné à la possibilité métaphysique d'une hallucination parfaite une valeur équivalente à celle du fait de l'illusion.

Une réponse possible, aujourd'hui adoptée de manière quasi-unanime, se trouve chez Price qui propose de distinguer au sein de l'argument de l'illusion un argument » phénoménologique » et un argument «causal ». En effet, tandis que le premier s'appuie sur un fait phénoménologique direct, à savoir l'expérience de l'illusion en tant que telle, l'argument causal s'appuie sur un fait physique indirect, à savoir l'existence de «certains processus, autres que la sensation mais contemporains de celle-ci» et qui «se produisent dans le système nerveux ${ }^{45}$. Ainsi, dans un cas l'indifférenciabilité de la perception et de l'illusion est dérivée d'une comparaison phénoménologique de ces expériences, dans l'autre l'indifférenciabilité est dérivée du fait que ces expériences possèdent les mêmes causes physiques. Si Price se réfère à ces deux versions de l'argument de l'illusion, dans le cas de l'hallucination seul l'argument causal peut être retenu. En effet, comme nous l'avons vu, l'argument de l'hallucination (à la différence de celui de l'illusion) ne peut être phénoménologique sans se contredire lui-même. C'est pourquoi le concept d'hallucination parfaite devra nécessairement être dérivé, par une expérience de pensée, des faits physiques impliqués dans la perception.

45. H. Price, Perception, Westport, Greenwood, 1981, p. 27. 
Si cet argument ne sera pas développé par Price, qui y voit un « idéalisme physiologique » qu'il n'hésite pas à qualifier de «stupide ${ }^{46}$, ce dernier est en revanche défendu et détaillé par Howard Robinson de la manière suivante :

«(1) Possibilité des hallucinations des philosophes. Il est théoriquement possible, en activant quelques processus cérébraux impliqués dans un type particulier de perception, de produire une hallucination qui soit subjectivement indifférenciable de cette perception ${ }^{47}$.

Comme le montre le caractère «théoriquement possible» de l'hallucination du philosophe, celle-ci reste pour Robinson une possibilité métaphysique. Cependant, cette possibilité métaphysique est ici inférée à partir de processus neuronaux qui sont eux-mêmes impliqués dans toute perception. Par suite, dans la mesure où l'on ne saurait nier l'existence de ces faits physiques, le philosophe de la perception semble bien contraint de rendre compte de l'hallucination parfaite inférée à partir de ces derniers. Inférence qui est loin d'aller de soi car, si personne ne va nier que certains processus cérébraux sont impliqués dans la perception, on ne saurait en conclure pour autant qu'ils suffisent à produire celle-ci. C'est pourquoi Robinson complète la proposition précédente par le principe suivant :

«(2) Même cause proximale, même effet immédiat. Il est nécessaire de décrire de la même manière les hallucinations et les expériences perceptives lorsqu'elles ont les mêmes causes neurales ${ }^{48}$.

La fonction de cette seconde proposition est ainsi de permettre de passer d'une relation d'implication entre processus neuronaux et expérience perceptive à une relation proprement causale. Ainsi, seuls les processus neuronaux font partie de la «cause proximale » de la perception, écartant tout autre élément (le corps du sujet, l'objet perçu, l'environnement, etc.) faisant partie seulement des «causes distales» qui ne participent qu'indirectement à l'expérience perceptive ${ }^{49}$. Une fois ce nouveau principe posé, rien n'empêche alors de concevoir une expérience qui serait subjectivement identique à une perception, car elle partage la même cause proximale que celle-ci, et pourtant indépendante de toute réalité

46. Ibid, p. 40.

47. H. Robinson, op. cit., p. 313.

48. Idem.

49. On notera que cette division entre cause «proximale» et cause «distale» ne va pas de soi, même dans un cadre strictement physicaliste. Ainsi, David Chalmers a montré la difficulté que représente toute tentative d'isoler un corrélat neuronal de la conscience (D. Chalmers, "What is a Neural Correlate of Consciousness ? , in Neural Correlates of Consciousness: Empirical and Conceptual Questions, Metzinger (éd.), Cambridge, MIT Press, 2000) ce que présuppose pourtant l'expérience de pensée en question. Néanmoins, notre propos n'est pas d'interroger la cohérence interne d'une telle expérience de pensée mais de montrer la faute logique que constitue son usage dans l'argument de l'hallucination. 
externe, dans la mesure où aucune cause distale n'est impliquée dans sa production. Autrement dit, il devient possible de déduire le concept d'hallucination parfaite des causes physiques de la perception.

\subsection{La perception comme « chose »}

Toute la question est alors de savoir si l'argument causal parvient réellement à penser l'hallucination sans rien dire de la perception. De fait, avant d'apparaître en philosophie, ces expériences de pensée étaient fréquemment utilisées dans l'étude empirique de l'hallucination et ont fait l'objet de nombreuses critiques qui mériteraient d'être étendues à la philosophie. C'est notamment le cas d'Henri Ey qui, dans son Traité des hallucinations, montre que cette «mythologie neurologique» introduit, implicitement, une certaine ontologie de l'expérience perceptive. Comme l'observe Ey, dire que l'hallucination est une «production » du cerveau ne revient pas à l'expliquer par une cause physique mais à en proposer une description métaphorique. Or le propre de l'image du cerveau "créateur » est qu'elle est indissociable d'une représentation de l'hallucination comme «chose». En effet, dès lors qu'on conçoit l'expérience hallucinatoire comme quelque chose que le cerveau produirait en dehors et en plus de luimême, cela signifie que, si celle-ci ne fait pas partie du monde, elle ne saurait davantage faire partie du sujet. Ainsi, on introduit subrepticement une représentation de l'hallucination comme « corps étranger ${ }^{50}$ qui flotterait entre le sujet et le monde. C'est pourquoi, sans nécessairement en avoir conscience, ces expériences de pensée nous amènent à concevoir l'hallucination "comme une chose, comme un être que les yeux, les oreilles de l'observateur [externe] pourraient peut-être un jour percevoir $»^{51}$. Par conséquent, ces expériences de pensée, qui relèvent davantage de la métaphore que du fait physique, impliquent une réification de l'hallucination.

Si Ey s'insurge, pour des raisons qui lui sont propres, contre cette conception de l'hallucination comme « chose», son constat peut être étendu a fortiori à la perception. En effet, rappelons-le, l'hallucination parfaite est créée à partir des processus neuronaux impliqués dans la perception, ce qui suppose que celle-ci à son tour est déjà conçue comme une «chose » produite par le cerveau. Ainsi, la possibilité métaphysique de l'hallucination parfaite ne peut être légitimée qu'au sein d'une ontologie de la perception comme «chose». Or, dans la mesure où l'argument philosophique de l'hallucination prétend déterminer la nature de la perception, il est nécessaire qu'il ne présuppose rien concernant cette dernière, ce qu'il échoue à faire dès lors qu'il conçoit la perception en ces termes. On pourrait alors objecter que le terme de «chose», comme tout terme transcendantal, est par essence

50. Ibid., p. 92.

51. Op. cit, p. 90. 
indéterminé de sorte qu'il ne dit rien de la perception. En réalité, dire d'une perception qu'elle est une «chose » c'est déjà exclure toute conception réaliste. En effet, le propre du réalisme naïf est de concevoir l'expérience perceptive comme «transparente » au sens où le contenu de la perception n'est rien d'autre que l'objet lui-même ${ }^{52}$. C'est ce qui le distingue non seulement de la théorie des sense-data qui conçoit la perception comme un objet mental (ou dans certains cas physique), mais aussi de l'intentionalisme représentationaliste qui pense la perception en termes de «contenu représentationnel ». Or dès lors qu'on conçoit la perception comme une « chose », même indéterminée, celle-ci ne peut être conçue autrement que comme un intermédiaire entre ce dont nous faisons l'expérience et le monde. Par suite, au sein de ce cadre théorique, la transparence de notre perception devient nécessairement une illusion puisque ce dont nous faisons l'expérience n'est jamais l'objet lui-même mais bien une «chose » venant s'interposer entre nous et lui. C'est pourquoi ces expériences de pensée reposant sur la «mythologie neurologique» de l'hallucination parfaite ne sont compatibles qu'avec une conception de la perception comme «représentation» voire comme «sense-data», c'est-à-dire avec une conception de la perception comme « chose ».

Ce qui apparaît alors de manière évidente, c'est le fait que l'argument de l'hallucination contient une prémisse implicite (la métaphore de la perception comme «chose ») qui correspond mot pour mot à ce qui est censé être sa conclusion. En effet, rappelons-le, le but de l'argumentation de l'hallucination est d'arriver à la proposition (4) d'après laquelle «les contenus des perceptions véridiques sont des images mentales et non des objets externes concrets $»^{53}$. Or une telle conception de la perception est déjà admise dès lors qu'on accepte l'idée d'un cerveau produisant les expériences perceptives. Par suite, le détour opéré par, d'une part, l'indifférenciabilité de l'hallucination et de la perception et, d'autre part, la présence de propriétés communes (dans les propositions (2) et (3)), se révèle parfaitement inutile puisque le concept d'hallucination parfaite est déjà contenu dans celui de perception. On peut donc dire que le concept de perception présupposé par cet argument n'est rien d'autre que celui d'une expérience perceptive qui ne dépend de la réalité que de manière indirecte, c'est-à-dire (pour reprendre l'expression d'Hyppolite Taine) une «hallucination vraie». En ce sens, l'argument de l'hallucination ne comporte aucune contradiction logique interne (contrairement à ce que soutient le disjonctivisme ${ }^{54}$ ), pour la simple

52. M.G.F. Martin, «The transparency of experience», Mind \& Language, 4, (2002), p. 376-425.

53. Op. cit.

54. Si nous ne pouvons développer ce point ici il est essentiel de noter que si l'identification de l'hallucination parfaite et de la perception par le conjonctivisme est tautologique, alors leur différenciation par le disjonctivisme est nécessairement contradictoire. En effet, le disjonctiviste adhère à la possibilité de l'hallucination parfaite (sans quoi il n'y a plus d'argument à réfuter) et ce faisant valide une conception représentationaliste de la perception tout en prétendant défendre un réalisme naïf. A ce titre la conception «épistémique négative » 
raison qu'il est purement tautologique et par suite incapable de démontrer quoique ce soit concernant la perception.

\section{Conclusion}

Notre propos était de montrer que le concept d'hallucination ne peut être pensé qu'à partir de celui de perception. Ce primat de la perception apparaît d'abord dans l'histoire de la psychopathologie dans la mesure où la différence introduite par le psychiatre entre «fausse hallucination» et «hallucination véritable» dépend de ce que ce dernier entend par «percevoir». Mais il n'en va pas autrement pour la philosophie dont le concept «d'hallucination parfaite » est aussi pensé à partir de la perception comme « chose » c'est-à-dire comme « hallucination vraie ». Cependant, à la différence de la psychopathologie, la philosophie semble avoir oublié ce primat de la perception dans la mesure où elle prétend déterminer en retour celle-ci à partir de l'hallucination. De ce fait, l'argument de l'hallucination possède une forme circulaire et tautologique qui, en tant que telle, ne semble pas pouvoir contribuer à notre connaissance de la perception.

Faut-il pour autant abandonner ce dernier? Si le concept d'hallucination parfaite (et l'argument causal sur lequel il repose) ne peut rien dire de la perception, il reste possible et souhaitable de l'abandonner pour se tourner vers l'expérience hallucinatoire elle-même. Par suite, s'il y a un argument de l'hallucination il ne peut être que phénoménologique et non causal. Cependant, et c'est là l'apport crucial de la psychopathologie à la philosophie, il serait naïf de croire que l'hallucination constitue un fait brut, ce qui est décrit comme une expérience hallucinatoire l'étant toujours à partir de ce que le sujet nomme percevoir. C'est pourquoi pour donner sens à l'argument phénoménologique de l'hallucination, il nous semble nécessaire de se demander non seulement comment l'expérience hallucinatoire nous apparaît mais, avant cela, ce qu'halluciner veut dire.

de l'hallucination défendue par Martin dans The Limits of Self-awareness (op. cit., p. 22) et On Being Alienated (op. cit., p. 2) est symptomatique de cette contradiction dans laquelle les réalistes naïfs s'enlisent dès lors qu'ils adoptent la théorie disjonctiviste. En quelques mots, la position de Martin consiste à soutenir que la seule propriété que l'on peut attribuer à l'hallucination est une propriété négative, à savoir celle d'être indifférenciable d'une perception, de sorte qu'on ne saurait attribuer d'élément commun à l'hallucination et la perception. Cette position a souvent été présentée comme étant dépourvue de sens, dans la mesure où il semble inconcevable qu'une expérience, même fausse, puisse être dépourvue de toute propriété positive. Cependant, ce non-sens est la seule conclusion à laquelle peut arriver tout réaliste naïf qui accepte le concept d'hallucination parfaite puisque reconnaitre la moindre propriété positive à cette dernière l'enferme nécessairement dans un cadre représentationaliste. Il nous semble toutefois plus simple et cohérent de refuser la prémisse de l'hallucination parfaite, plutôt que de l'accepter pour ensuite tenter de la nier comme le fait le disjonctiviste. 